\title{
Prolidase Deficiency in Very Early Onset Inflammatory Bowel Disease (VEO-IBD)
}

\author{
Manoj Madhusudan ${ }^{1} \cdot$ Srinivas Sankaranarayanan ${ }^{1} \cdot$ Ramkumar Ramamoorthy $^{2} \cdot$ Deenadayalan Munirathnam $^{3}$. \\ Meena Sivasankaran ${ }^{3}$
}

Received: 6 October 2020 / Accepted: 23 December 2020/ Published online: 9 January 2021

(C) Dr. K C Chaudhuri Foundation 2021

To the Editor: A 4-y-old boy 2nd born to 3rd degree consanguineous marriage, presented with failure to thrive, developmental delay and recurrent episodes of nonbloody diarrhea since $1 \mathrm{y}$ of age. He had multiple episodes of ear discharge and recurrent skin ulcers involving the lower limbs.

He had frontal bossing, sparse hypopigmented hair, pedal edema and generalized scaling with nonhealing ulcers in the lower limbs. Systemic examination was normal. Investigations revealed microcytic hypochromic anemia, thrombocytopenia, hypoalbuminemia and elevated C-reactive protein (CRP). Liver and renal parameters were unremarkable. Evaluation for infection, immunodeficiency and celiac disease were negative.

Ultrasound abdomen showed mural thickening of the entire colon. Colonoscopy revealed complete loss of normal vascular pattern with superficial erosions of the entire colon and biopsies revealed a crypt destructive colitis which raised the possibility of very early onset inflammatory bowel disease (VEO-IBD). He was started on steroids and his IBD underwent clinical remission. He has remained under follow-up for one year and is doing well on alternate day low dose steroids, though he continues to have recurrent skin ulcers and failure to thrive.

In view of parental consanguinity and the presence of facial dysmorphism, recurrent ear infections, and skin ulcers in a child with VEO-IBD, next generation sequencing was done which revealed a novel homozygous mutation in Exon 12 of PEPD gene [c.825delC (p.Phe275LeufsTer46)], which was

Srinivas Sankaranarayanan

dr.srinis@gmail.com

1 Department of Pediatric Gastroenterology, Kanchi Kamakoti CHILDS Trust Hospital, 12 A, Nageswara Road, Nungambakkam, Chennai, Tamil Nadu 34, India

2 Department of Pediatric Dermatology, Kanchi Kamakoti CHILDS Trust Hospital, Chennai, Tamil Nadu, India

3 Department of Pediatric Hematology, Kanchi Kamakoti CHILDS Trust Hospital, Chennai, Tamil Nadu, India pathogenic for prolidase deficiency (PD). Sanger sequencing confirmed both parents as heterozygous carriers of the same mutation.

PD is a very rare genetic disorder with fewer than 100 cases reported [1]. It manifests with a variety of clinical features with facial dysmorphism and dermatological manifestations being the most common [1]. Gastrointestinal manifestations with a VEO-IBD like phenotype are rare and have variable response to medical treatment $[2,3]$.

While an infant with PD having colitis responded to steroids [2], another 5-y-old boy with PD and a Crohn's disease-like phenotype did not respond to steroids, methotrexate and rituximab [3]. In the present child the colonoscopy suggested features suggestive of ulcerative colitis which improved with steroids.

This case is presented for the extreme rarity (Incidence 1-2/ $1,000,000$ persons) [3], and to demonstrate the role of molecular genetics in children with VEO-IBD.

\section{Compliance with Ethical Standards}

Conflict of Interest None.

\section{References}

1. Spodenkiewicz M, Spodenkiewicz M, Cleary M, et al. Clinical genetics of prolidase deficiency: an updated review. Biol (Basel). 2020;9(5):108.

2. Kuloglu Z, Kansu A, Serwas N, et al. Inflammatory bowel diseaselike phenotype in a young girl with prolidase deficiency: a new spectrum of clinical manifestation. Genet Couns. 2015;26(2):20511.

3. Rizvi SA, Elder M, Beasley G. A novel manifestation of prolidase deficiency in a toddler diagnosed with very-early-onset crohn disease. J Pediatr Gastroenterol Nutr. 2019;69(3):e89-90.

Publisher's Note Springer Nature remains neutral with regard to jurisdictional claims in published maps and institutional affiliations. 